\title{
NATIONAL HEALTH AND MEDICAL RESEARCH COUNCIL INFANT FEEDING GUIDELINES FOR HEALTH WORKERS, 2003
}

In 2003, the National Health and Medical Research Council updated Australia's Infant Feeding Guidelines for Health Workers and incorporated them into the Dietary guidelines for children and adolescents in Australia. ${ }^{1}$ The new Infant Feeding Guidelines recommend that as many infants as possible in Australia be exclusively breastfed until six months of age and that mothers continue breastfeeding until their infants are 12 months of age, and beyond if both mother and infant wish.

'All health workers have an obligation to promote breastfeeding in the community and to ensure that best practice in breastfeeding is followed.'

The Infant Feeding Guidelines provide information to help health professionals encourage, support and promote breastfeeding. They:

- endorse breastfeeding as the normal way to feed all infants;

- recommend ways that community health, primary health care services, hospitals and workplaces can help to increase breastfeeding initiation rates and duration;

- discuss the few contra-indications for breastfeeding, such as the mother taking drugs, and provide strategies to identify these contra-indications and reduce their impact;

- provide guidelines for appropriate support and advice when an infant is not receiving breastmilk;

- provide recommendations for infant feeding, including the use of fluids other than breastmilk, the timing for introducing solid food, caring for infants' food and nutrition in the second year of life;

- discuss other aspects of infant nutrition such as food allergies, colic, constipation, dietary fat, dental caries, diarrhoeal disease, iron deficiency anaemia, vegetarian diets, feeding low birthweight infants and foods not suitable for infants.

Health workers will find the following particularly helpful:

- a glossary of terms associated with infant feeding and breastfeeding;

- descriptions of initiatives such as the WHO International Code of Marketing of Breastmilk Substitutes (including an interpretation of the WHO Code for health workers in Australia, health workers' responsibilities under the Code and how to report a breach of the Code), the Baby Friendly Hospital Initiative and the National Breastfeeding Strategy;

- a summary of the health benefits of breastfeeding for mother and infant;

- a description of the physiology of breastfeeding and normal infant behaviour;

- practical strategies for health workers to assist with initiation, establishment and maintenance of breastfeeding among their clients (including strategies for the 'early days' and common problems and their management);

- guidelines for expressing and storing breastmilk (particularly useful for supporting working mothers);

- the need for informed consent for complementary feeds in hospital;

- Australian nutrition and breastfeeding resources and websites.

Other items of interest include:

- a discussion of measurement of breastfeeding rates;

- information about the Innocenti Declaration on the Protection, Promotion and Support of Breastfeeding and the Royal Australian College of General Practitioners Breastfeeding Position Statement;

- a description of the composition of human milk.

\section{REFERENCE}

1. National Health and Medical Research Council. Dietary guidelines for children and adolescents in Australiaincorporating the infant feeding guidelines for health workers. Commonwealth of Australia, 2003. ISBN Print:1864961538 Online: 1864961473.

Dietary guidelines for children and adolescents in Australia is sold through AusInfo Government Info Bookshops. The toll-free number for purchases is 132 447. The Infant Feeding Guidelines are included on the CD enclosed with this issue of the Bulletin, and can also be found on the Internet at www.nhmrc.gov.au/publications/pdf/n34.pdf . 중 